\title{
Análise das características do vento sobre a Floresta Nacional do Tapajós, Pará, Brasil
}

Analysis of wind characteristics on the Tapajós National Forest, Pará, Brazil

\section{Raphael Tapajós ${ }^{1,2}$, Rodrigo da Silva ${ }^{2}$, Wilderclay Machado ${ }^{1,3}$, Raoni Santana ${ }^{1}$, Roseilson do Vale ${ }^{1}$, Miércio Ferreira-Junior ${ }^{3}$}

rpablotapajos@gmail.com; rsilvf@yahoo.com.br; wilderclay@yahoo.com.br; raoniass@gmail.com; roseilsondovale@gmail.com; miercio_junior@yahoo.com.br

${ }^{1}$ Mestre, Ciências Atmosféricas, Universidade Federal do Oeste do Pará, Santarém, PA, Brasil ${ }^{2}$ Doutorando, Programa de Pós-Graduação em Sociedade, Natureza e Desenvolvimento, Universidade Federal do Oeste do Pará, Santarém, PA, Brasil

${ }^{3}$ Doutor, Ciências Atmosféricas, Universidade Federal do Oeste do Pará, Santarém, PA, Brasil

\begin{abstract}
Resumo
A circulação atmosférica da região do Baixo Tapajós apresenta características únicas devido a influência de florestas, campos agrícolas, rios e áreas urbanas. O estudo, no que se refere ao campo de vento em dois pontos distindos da Floresta Nacional do Tapajós (FNT), mostra que há diferenças significativas na direção do vento nesses pontos. A identificação da canalização Norte e Sul, e ventos de Oeste e quantificação das mesmas, dão indicativos importantes para estudos da influência de áreas adistintas sobre FNT. Os resultados mostram que as ocorrências de canalização e ventos de Oeste, são mais presentes no período chuvoso e ocorrem mais durante o dia. Além disso, na estação de Jamaraquá, mais próximo do Rio Tapajós, as influências de canalização e ventos de Oeste são maiores, o que pode ser devido não apenas a proximidade do rio mas também aos aspectos topográficos.
\end{abstract}

Palavras-chave: Floresta Nacional do Tapajós (FNT), Vento, Canalização Norte e Sul, Brisa, Jamaraquá e Km67.

\begin{abstract}
The atmospheric circulation of the Tapajós River region has unique characteristics due to influences of forests, agricultural fields, rivers and urban areas. The study with regard to the wind field in two different points in National Forest Tapajós (FNT) shows that there are significant differences in wind direction at those points. Identifying and quantify of the North and South canalization, and westerly winds, give important indications for studying the influence of different areas on the FNT. The results show that the occurrences of drains and west winds are more present during the rainy season and occur more during the day. Furthermore, in Jamaraquá station nearest to the River, the influence of channeling and west winds are greater, which can be due not only proximity of river but also the topographical features.
\end{abstract}

Keywords: National Forest Tapajós (FNT), wind, canalization North and South, Breeze, Jamaraquá and Km67. 


\section{Introdução}

A região Amazônica é formada por um grande mosaico de diferentes coberturas superficiais, como florestas, grandes rios, campos agrícolas, áreas degradadas e cidades. Na região do Baixo Tapajós no Oeste do Pará, investigada por apresentar essas características, mostra-se muito interessante devido ter peculiaridades na circulação atmosférica.

A região de confluência dos Rios Tapajós e Amazonas, apresenta climatologia única como descrito por Cohen et al. (2014), Fitzjarrald et al. (2008) e Silva Dias et al. (2004). Estes mostraram que a região é dominada por regimes de circulação atmosférica diferenciada, formação de nuvens, ocorrência de brisa de rio, influenciando na distribuição espacial e horária da de chuva. Além disso, estudos como de Lu et al. (2005) sugerem que uma análise detalhada da influência da circulação sobre as medidas de fluxos de massa e energia na Floresta Nacional do Tapajós (FNT), que ocorre a mais de uma década.
Com isso, este trabalho tem como objetivo fazer uma análise, a partir de dados superficiais, do regime de vento sobre a FNT, levando em consideração a ocorrência de canalização Norte-Sul, Sul-Norte e Brisa do Rio Tapajós.

\section{Metodologia}

Os dois pontos de medidas ficam dentro da (FNT), na cidade de Belterra/PA (Figura 1). O primeiro, a 64 metros de altura (30 acima da copa das árvores) em uma torre micrometeorológica próximo a BR-163, Santarém-Cuiabá, no Km67, (-2,851 ${ }^{\circ}$; $\left.54.967^{\circ}\right)$; o segundo, a 10 metros de altura em uma estação meteorológica, na comunidade de Jamaraquá (JAM) às margens do rio Tapajós ($\left.2,807^{\circ} ;-55,036^{\circ}\right)$, ambos pertencentes ao Programa de Grande Escala Biosfera Atmosfera na Amazônia (LBA), e fazem o monitoramento da região do Baixo Tapajós a mais de uma década. A escolha dos pontos distintos deverá mostrar padrões de circulação local e influências regionais, como a presença do rio Tapajós à oeste.

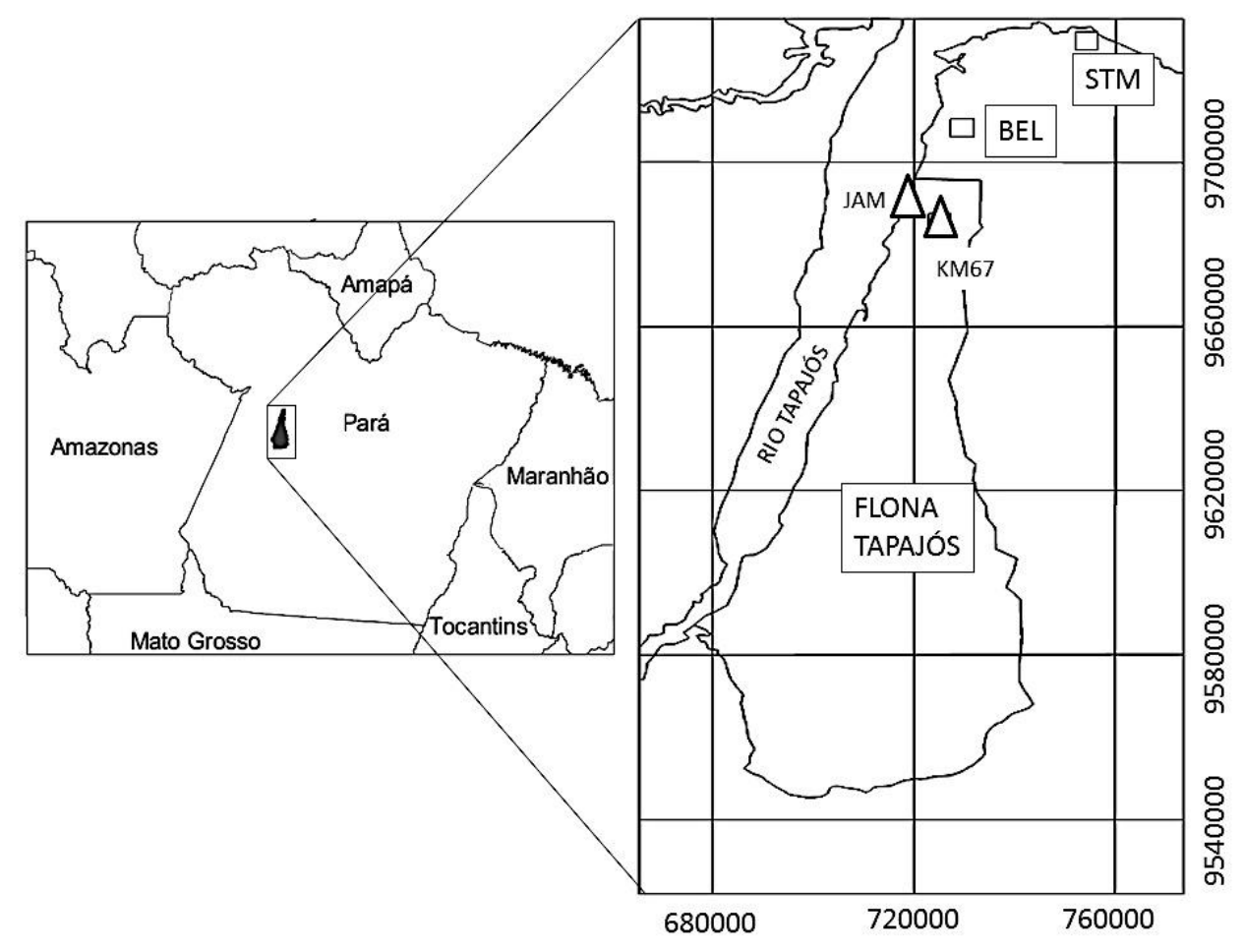

Figura 1. Localização dos pontos de medida em Jamaraquá (JAM) e Km67. Destaque para as cidades de Santarém (STM), Belterra (BEL) e a FLT. 
Para estudo da circulação local foram analisados dados (médias horárias) de velocidade e direção do vento, para o ano de 2002, medidos por um anemômetro sônico 3D (CSAT-3D, Campbell Sci.) no km67 e um anemômetro de copo (034A, Met One) em Jamaraquá.

Como na região ocorre a canalização dos ventos alísios devido a topografia regional (LU et al., 2005), e também a ocorrência de brisa do rio Tapajós, fez-se a classificação da direção do vento em quatro grandes setores: Norte $(\mathrm{N})$, Sul (S), Leste (L) e Oeste (O) e em análise mais detalhada em subsetores: Nordeste (NE), Sudeste(SE), Noroeste (NO) e Sudoeste (SO).

\section{Resultados e discussões}

As análises mostram que a região da FNT apresenta uma circulação peculiar, apresentando o padrão de ventos alísios de Leste (aproximadamente 75\%), mas também a ocorrência de ventos de Norte $(12,9 \%$ e $11,4 \%)$, de Sul $(6,8 \%$ e $8,7 \%)$ de Oeste $(5,3 \%, 4,1 \%)$ como descrito na tabela 1 .

Tabela 1. Descrição geral da velocidade e direção do vento

\begin{tabular}{|c|c|c|c|c|c|c|c|c|c|c|c|c|c|c|c|}
\hline \multirow{2}{*}{$\frac{\text { Local }}{\mathrm{D}}$} & \multicolumn{7}{|c|}{$\mathrm{Km} 67$} & \multicolumn{8}{|c|}{ Jamaraquá } \\
\hline & $\mathrm{V}$ & $T$ & $0-1$ & $1-2$ & $2-3$ & $3-4$ & $4-5$ & D & $\mathrm{V}$ & $\mathrm{T}$ & $0-1$ & $1-2$ & $2-3$ & $3-4$ & $4-5$ \\
\hline & $\mathrm{m} / \mathrm{s}$ & {$[\%]$} & {$[\%]$} & {$[\%]$} & {$[\%]$} & {$[\%]$} & {$[\%]$} & & $\mathrm{m} / \mathrm{s}$ & {$[\%]$} & {$[\%]$} & {$[\%]$} & {$[\%]$} & {$[\%]$} & {$[\%]$} \\
\hline $\mathbf{N}$ & 2,0 & 12,9 & 0,6 & 5,6 & 6,2 & 0,6 & 0,01 & $\mathbf{N}$ & 1,5 & 11,4 & 2,6 & 5,9 & 2,5 & 0,3 & 0,03 \\
\hline L & 2,3 & 74,9 & 1,5 & 21,2 & 43,5 & 8,4 & 0,4 & $\mathbf{L}$ & 2,0 & 75,8 & 13,7 & 23,5 & 27,2 & 10 & 1,4 \\
\hline$S$ & 1,6 & 6,8 & 1,4 & 3,5 & 1,8 & 0,1 & 0,01 & $S$ & 0,9 & 8,7 & 5,7 & 2,2 & 0,7 & 0,1 & \\
\hline $\mathrm{O}$ & 1,6 & 5,3 & 1,1 & 2,9 & 1,2 & 0,1 & & $\mathrm{O}$ & 1,7 & 4,1 & 0,7 & 2,3 & 0,9 & 0,2 & 0,03 \\
\hline $\mathrm{T}$ & 2,2 & 100 & 4,6 & 33,1 & 52,7 & 9,2 & 0,4 & $T$ & 1,8 & 100 & 22,6 & 33,9 & 31,3 & 10,7 & 1,4 \\
\hline
\end{tabular}

Legenda: D - direção; V -Velocidade; N - Norte; L - Leste; S - Sul; O - Oeste; T - Total

As velocidades são características para cada local. No Km67 mais da metade dos dados $(52,7 \%)$ ficaram entre 2 e $3 \mathrm{~m} / \mathrm{s}$, em JAM a maior parte das ocorrências são divididas entre $0-3 \mathrm{~m} / \mathrm{s}$. Para os ventos canalizados de $\mathrm{S}$ e $\mathrm{O}$ os valores de velocidade apresentam-se sempre baixos, entre 0 e $3 \mathrm{~m} / \mathrm{s}$, sendo a maior predominância entre 1-2 m/s no Km67. Na canalização $\mathrm{N}$ os valores predominantes são entre 1 e $3 \mathrm{~m} / \mathrm{s}$. Vale salientar a grande ocorrência de ventos de Nordeste (NE), sendo cerca de 35,8\% das medidas dos ventos alísios no Km67, e Sudeste (SE), cerca de $27 \%$, em JAM, e que os ventos de Oeste (brisa do rio Tapajós), é predominantemente de Noroeste (NO) nos dois sites, fatos esses que deve acontecer devido à topografia regional como citado por Lu et al., (2005).

Outro resultado importante que foi encontrado é que a ocorrência de canalização $\mathrm{N}$ e ventos de L não apresentaram diferenças muito grandes entre o período da noite e do dia no Km67, sendo que durante o dia a ocorrência de ventos de $\mathrm{O}$ aumentou para aproximadamente $7 \%$ e as canalizações $\mathrm{N}$ e $\mathrm{S}$ para $14 \%$ e $7 \%$ respectivamente, e os ventos de Leste mantiveram o mesmo padrão geral. Porém, em JAM as mudanças foram maiores, com aumento da canalização $\mathrm{N}$ para $20 \%$ e ventos de $\mathrm{O}$ para $7 \%$.

Os valores de velocidade, como esperado, foram maiores durante o dia para os dois sítios, com maior ocorrência entre 1 e $4 \mathrm{~m} / \mathrm{s}$ para os ventos de L. Ventos de $\mathrm{N}$ e $\mathrm{S}$ ficaram entre $1 \mathrm{e}$ $3 \mathrm{~m} / \mathrm{s}$. No período noturno observou-se cerca de $75 \%$ e $88 \%$ das ocorrências de ventos de Leste para Km67 e JAM, respectivamente. No Km67 durante a noite teve diminuição tanto das canalizações $\mathrm{N}$ e ventos de $\mathrm{O}$ e aumento da canalização Sul, passando de 5,8\% para 8,1\% das ocorrências. Em Jamaraquá, a diminuição foi brusca, chegando a valores de $2 \%$ para canalização $\mathrm{N}$, e $0,7 \%$ de $\mathrm{O}$, porém com aumento de ventos $\mathrm{S}(9,8 \%)$. 
Em análise sazonal (tabela 2), observou-se diferenças entre os padrões de circulação, com maiores ocorrências de canalizações $\mathrm{S}, \mathrm{N}$ e ventos de Oeste no período de chuvas, para ambos os locais de medida. No Km67 a canalização $\mathrm{S}$ diminuiu de $14,0 \%$ (chuvoso) para $11,9 \%$ (seco), a canalização $S$ de $8,1 \%$ para $5,6 \%$ e vento de Oeste, de 6,1\% para $4,6 \%$ das ocorrências. Em Jamaraquá a diferença maior foi a diminuição na canalização $S$, de $12,5 \%$ para $7,2 \%$.

Tabela 2. Descrição sazonal da direção e velocidade do vento

\begin{tabular}{|c|c|c|c|c|c|c|c|c|c|c|c|c|c|c|c|}
\hline \multicolumn{8}{|c|}{ Km67-CHUVOSO (Janeiro à Junho) } & \multicolumn{8}{|c|}{ Km67 - SECO (Julho à Dezembro) } \\
\hline $\mathbf{D}$ & $\mathbf{V}$ & $\mathbf{T}$ & $0-1$ & $1-2$ & $2-3$ & $3-4$ & $4-5$ & D & $\mathbf{V}$ & $\mathbf{T}$ & $0-1$ & $1-2$ & $2-3$ & $3-4$ & $4-5$ \\
\hline & $\mathrm{m} / \mathrm{s}$ & [\%] & {$[\%]$} & [\%] & [\%] & [\%] & [\%] & & $\mathrm{m} / \mathrm{s}$ & {$[\%]$} & {$[\%]$} & [\%] & {$[\%]$} & {$[\%]$} & [\%] \\
\hline $\mathbf{N}$ & 1,9 & 14,0 & 0,9 & 7,1 & 5,3 & 0,7 & 0,0 & $\mathbf{N}$ & 2,1 & 11,9 & 0,2 & 4,1 & 7,1 & 0,5 & \\
\hline $\mathbf{L}$ & 2,1 & 71,8 & 2,2 & 27,4 & 36,9 & 5,1 & 0,2 & $\mathbf{L}$ & 2,5 & 77,9 & 0,8 & 15,1 & 50,1 & 11,6 & 0,5 \\
\hline$S$ & 1,6 & 8,1 & 1,6 & 4,4 & 2,0 & 0,1 & 0,0 & $S$ & 1,6 & 5,6 & 1,2 & 2,7 & 1,6 & 0,2 & \\
\hline $\mathrm{O}$ & 1,6 & 6,1 & 1,3 & 3,2 & 1,4 & 0,1 & & $\mathrm{O}$ & 1,6 & 4,6 & 1,0 & 2,6 & 0,9 & 0,1 & \\
\hline $\mathrm{T}$ & 2,0 & 100 & 6,0 & 42,1 & 45,6 & 6,0 & 0,3 & $T$ & 2,3 & 100 & 3,2 & 24,4 & 59,7 & 12,3 & 0,5 \\
\hline \multicolumn{8}{|c|}{ Jamaraquá - CHUVOSO (Janeiro à Junho) } & \multicolumn{8}{|c|}{ Jamaraquá - SECO (Julho à Dezembro) } \\
\hline $\mathbf{D}$ & $\mathbf{V}$ & $T$ & $0-1$ & $1-2$ & $2-3$ & $3-4$ & $4-5$ & $\mathbf{D}$ & $\mathbf{V}$ & $T$ & $0-1$ & $1-2$ & $2-3$ & $3-4$ & $4-5$ \\
\hline & $\mathrm{m} / \mathrm{s}$ & {$[\%]$} & {$[\%]$} & [\%] & {$[\%]$} & {$[\%]$} & {$[\%]$} & & $\begin{array}{c}\mathrm{m} / \\
\mathrm{s}\end{array}$ & {$[\%]$} & {$[\%]$} & {$[\%]$} & {$[\%]$} & {$[\%]$} & [\%] \\
\hline $\mathbf{N}$ & 1,4 & 11,8 & 2,8 & 7,5 & 1,5 & & & $\mathrm{~N}$ & 1,6 & 11,3 & 2,5 & 5,3 & 2,9 & 0,5 & 0,1 \\
\hline $\mathbf{E}$ & 1,5 & 70,8 & 22,6 & 27,1 & 18,4 & 2,3 & 0,3 & $E$ & 2,2 & 77,8 & 10,1 & 22,1 & 30,7 & 13,1 & 1,8 \\
\hline $\mathrm{S}$ & 0,8 & 12,5 & 9,1 & 2,7 & 0,6 & & & $S$ & 1 & 7,2 & 4,3 & 2 & 0,7 & 0,1 & \\
\hline $\mathrm{O}$ & 1,6 & 4,9 & 0,9 & 2,7 & 1,1 & 0,2 & & $\mathrm{O}$ & 1,7 & 3,8 & 0,6 & 2,1 & 0,8 & 0,2 & 0,1 \\
\hline $\mathrm{T}$ & 1,4 & 100 & 35,5 & 40,1 & 21,7 & 2,5 & 0,3 & $\mathrm{~T}$ & 2,0 & 100 & 17,6 & 31,5 & 35,2 & 13,9 & 2,0 \\
\hline
\end{tabular}

Os valores de velocidade são menores no período chuvoso, com predominância entre $1 \mathrm{e}$ $3 \mathrm{~m} / \mathrm{s}$ para os ventos de leste e canalizações, e ventos de oeste com predominância entre 1 e 2 $\mathrm{m} / \mathrm{s}$, para os dois sítios, mas em Jamaraquá, há uma grande ocorrência de ventos fracos de 0-1 $\mathrm{m} / \mathrm{s}(35 \%)$. Para o período de estiagem, a maior parte das ocorrências de vento $L$ e canalização $\mathrm{N}$ são de 2 a $3 \mathrm{~m} / \mathrm{s}$, já as canalizações $S$ e ventos de Oeste a maior incidência de ventos entre 0 e $2 \mathrm{~m} / \mathrm{s}$.

A análise integrada dos dados mostra que a ocorrência de brisa do Rio e canalização N é predominante em ambos os sítios no período chuvoso, mas com incidência maior em JAM, podendo ou não ocorrer conjuntamente no Km67. Os horários de início da canalização norte e ventos de oeste são distintos para as duas localidades, sendo que em Jamaraquá iniciam mais cedo, entre 08:00 e 11:00hs, e no
Km67 entre 13:00 e 15:00hs. Os momentos em que a direção do vento começa a normalizar para leste ou nordeste é as 18:00hs em Jamaraquá e Km67, porém no Km67 pode chegar até as 21:00hs.

Notou-se também que antes do vento mudar de sua direção predominante (L), nos dois locais, existe decréscimo linear na velocidade do vento para abaixo de $1 \mathrm{~m} / \mathrm{s}$. Logo em seguida o vento se mantem entre 1 e $2 \mathrm{~m} / \mathrm{s}$ até diminuir novamente e voltar aos padrões normais de leste/nordeste e com velocidades entre 2 e $3 \mathrm{~m} / \mathrm{s}$.

\section{Conclusões}

Os padrões comportamentais do vento nos dois sítios analisados na FLONA Tapajós mostram-se distintos em alguns aspectos importantes, como na ocorrência 
de canalização e vento de Oeste (Brisa do rio Tapajós). Vale ressaltar que os ventos de Oeste tem predominância de Noroeste, reforçando que a presença da brisa do rio pode estar associada a canalização Norte e que estes processos ocorrem principalmente durante o dia e no período chuvoso.

Por fim, serão necessários estudos mais detalhados, dividindo em mais subsetores de campo de vento e análise de variáveis importantes como radiação, temperatura, umidade específica e até mesmo fluxo de $\mathrm{CO}_{2}$, correlacionando-as com os padrões de vento, visto que o site do Km67 é cercado de áreas distintas, e pode estar captando influência das mesmas.

\section{Agradecimentos}

À Coordenação de Aperfeiçoamento de Pessoal de Nível Superior (CAPES) pela bolsa de auxílio a pesquisa e ao Laboratório de Física e Química da Atmosfera (UFOPA) pelo apoio logístico.

\section{Referências}

Cohen, C. P. J.; Fitzjarrald, D. R.; D'oliveira, F. A. F.; Saraiva, I.; Barbosa, I. R. S.; Gandu A. W. ; Kuhn, P. A. Radar-Observed Spatial and Temporal Rainfall Variability near The Tapajós-Amazon Confluence. Revista Brasileira de Meteorologia, v. 29, n. esp., 23 30, 2014.

Fitzjarrald, D. R.; Sakai, R. K.; Moraes, O. L.; Cosme De Oliveira, R.; Acevedo, O. C.; Czikowsky, M. J; Beldini, T. Spatial and Temporal rainfall variability near the Amazon-Tapajós Rivers Confluence. Journal of Geophysical Research, vol. 113, 2008.

Lu, L.; Denning, A. S.; Silva-Dias, M. A.; Silva-Dias, P.; Longo, M.; Freitas, S. R.; Saatchi, S. Mesoscale circulations and atmospheric $\mathrm{CO}_{2}$ variations in the Tapajós Region, Pará, Brazil. Journal of Geophysical Research, vol. 110, 2005.
Silva Dias, M. A. F.; Silva Dias P. L.; Longo M.; Fitzjarrald D. R.; Denning A. S. River breeze circulation in eastern Amazonia: observations and modelling results. Theoretical and Applied Climatology, vol. 78(1), p. 111-121, 2004. 